\title{
EL DOCENTE Y LAS INTELIGENCIAS MÚLTIPLES
}

Silvia Luz de Luca

Docente Escuela rural, Argentina.

Este mundo nunca ha sido tan desigual en las oportunidades que brinda y tan igualitario en las costumbres que impone.

Eduardo Galeano

\section{INTRODUCCIÓN}

Este trabajo surgió a partir del año en que tuve como alumno a Luciano, un ser especialísimo. Bueno, dulce, cariñoso, servicial, muy hábil con las manos, con gran pasión por la naturaleza, la vida y el trabajo rural, pero con enormes dificultades para resolver situaciones lógico-matemáticas, extraer ideas principales de un texto, leer, redactar.

Me pregunté una y mil veces qué o cómo hacer para que aprendiera esos temas. Probé con las más variadas actividades, leí toda clase de bibliografía de la biblioteca escolar, comprada o prestada, hasta que me topé con las Inteligencias Múltiples. El tema me atrapó y decidí investigar más. Entonces quise volcarlo en un escrito para compartirlo con otros docentes que como yo, se encuentran ante situaciones similares y no tienen un equipo de profesionales a quien acudir en busca de ayuda.

Desgraciadamente Luciano ya no es más mi alumno, como la edad se lo permite, tiene 16 años, trabaja de día y concurre, por ahora, a la escuela nocturna para obtener su certificado de terminación de estudios primarios. No sé si hubiese podido ayudarlo, pero seguramente lo hubiese comprendido más y tal vez los dos nos hubiésemos sentido mejor.

\section{2. ¿DE QUÉ SE TRATA?}

En 1979 Howard Gardner, como investigador de Harvard, recibió el pedido de un grupo filantrópico holandés, la Fundación Bernard Van Leer, de dedicarse a investigar el potencial humano. A pesar de que Gardner ya había estado pensando en el concepto de "muchas clases de mentes" desde por lo menos mediados de la década del setenta, la publicación de su libro Frames of Mind (Estructuras de la mente) en 1983 marcó el nacimiento efectivo de la teoría de las inteligencias múltiples:

"En mi opinión, la mente tiene la capacidad de tratar distintos contenidos, pero resulta en extremo improbable que la capacidad para abordar un contenido permita predecir su facilidad en otros campos. En otras palabras, es de esperar que el genio ( $y$ a posteriori, el desempeño cotidiano) se incline hacia contenidos particulares: los seres humanos han evolucionado para mostrar distintas inteligencias y no para recurrir de diversas maneras a una sola inteligencia flexible."

(Gardner ,Estructuras de la Mente, 1994: 11)

"La teoría de las inteligencias múltiples puede describirse de la manera más exacta como una filosofía de la educación, un actitud hacia el aprendizaje, o aún como un meta-modelo educacional en el espíritu de las ideas de John Dewey sobre la educación progresiva. No es un programa de técnicas y estrategias fijas. De este modo, ofrece a los educadores una oportunidad muy amplia para adaptar de 
manera creativa sus principios fundamentales a cualquier cantidad de contextos educacionales". (Armstrong, Las inteligencias múltiples en el aula -12)

"Desde mi punto de vista, la esencia de la teoría es respetar las muchas diferencias que hay entre los individuos; las variaciones múltiples de las maneras como aparecen; los distintos modos por los cuales podemos evaluarlos, y el número casi infinito de modos en que estos pueden dejar una marca en el mundo". (Gardner, prólogo de Las inteligencias múltiples en el aula de Armstrong.)

La orientación crítica de Gardner hacia el concepto tradicional de inteligencia, está centrada en los siguientes puntos:

- La inteligencia ha sido normalmente concebida dentro de una visión uniforme y reductiva, como un constructo unitario o un factor general.

- La concepción dominante ha sido que la inteligencia puede ser medida en forma pura, con la ayuda de instrumentos estándar.

- Su estudio se ha realizado en forma descontextualizada y abstracta, con independencia de los desafíos y oportunidades concretas, y de factores situacionales y culturales.

- Se ha pretendido que es una propiedad estrictamente individual, alojada sólo en la persona, y no en el entorno, en las interacciones con otras personas, en los artefactos o en la acumulación de conocimientos.

Estamos acostumbrados a pensar en la inteligencia como una capacidad unitaria o como abarcativa de varias capacidades. Sin embargo, en oposición a esos enfoques de perfil más bien reduccionista, Gardner propone un enfoque de inteligencias múltiples. Se trata de un planteamiento sugerente, y acaso también provocativo, que permite problematizar sobre el fenómeno de la inteligencia más allá del universo de lo cognitivo.

Para este autor una inteligencia es la "capacidad de resolver problemas o de crear productos que sean valiosos en uno o más ambientes culturales", (1994; 10). Lo sustantivo de su teoría consiste en reconocer la existencia de ocho inteligencias diferentes e independientes, que pueden interactuar y potenciarse recíprocamente. La existencia de una de ellas, sin embargo, no es predictiva de la existencia de alguna de las otras.(1)

Al definir la inteligencia como una capacidad Gardner la convierte en una destreza que se puede desarrollar. Gardner no niega el componente genético.

Todos nacemos con unas potencialidades marcadas por la genética. Pero esas potencialidades se van a desarrollar de una manera o de otra dependiendo del medio ambiente, nuestras experiencias, la educación recibida, etc.

Ningún deportista de elite llega a la cima sin entrenar, por buenas que sean sus cualidades naturales. Lo mismo se puede decir de los matemáticos, los poetas, o de la gente emocionalmente inteligente.

Howard Gardner añade que igual que hay muchos tipos de problemas que resolver, también hay muchos tipos de inteligencia. Hasta la fecha Howard Gardner y su equipo de la universidad de Harvard han identificado ocho tipos distintos: 


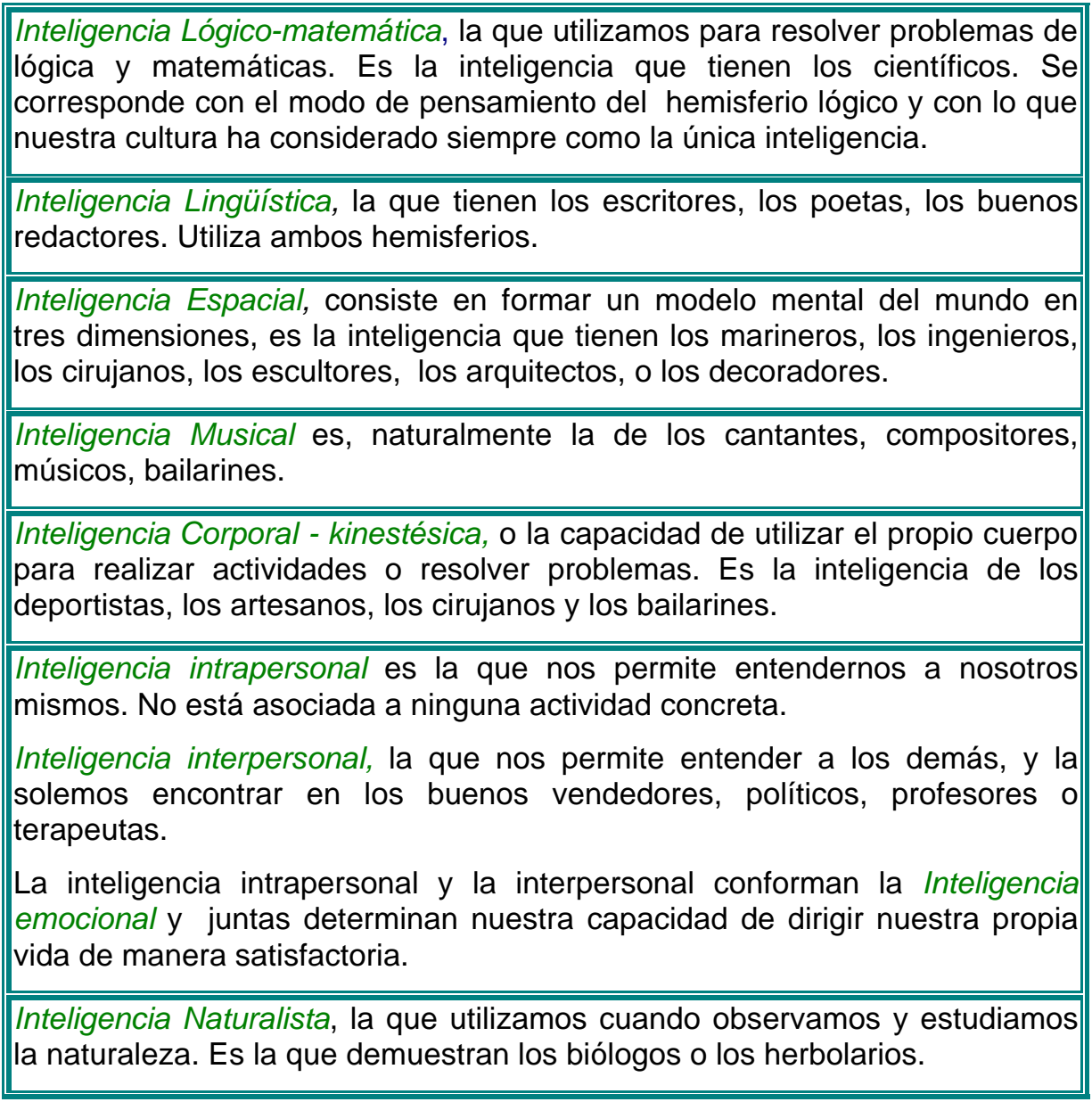

Naturalmente todos tenemos las ocho inteligencias en mayor o menor medida, (tal y como explica Fernando Lapalma en este artículo). Al igual que con los estilos de aprendizaje no hay tipos puros, y si los hubiera les resultaría imposible funcionar. Un ingeniero necesita una inteligencia espacial bien desarrollada, pero también necesita de todas las demás, de la inteligencia lógico matemática para poder realizar cálculos de estructuras, de la inteligencia interpersonal para poder presentar sus proyectos, de la inteligencia corporal - kinestésica para poder conducir su coche hasta la obra, etc.

Si la inteligencia es el conjunto de capacidades que nos permite resolver problemas o fabricar productos valiosos en nuestra cultura, la inteligencia emocional es el conjunto de capacidades que nos permite resolver problemas relacionados con las emociones. Con nuestras emociones (inteligencia intrapersonal) y con las de los demás (inteligencia interpersonal).

Daniel Goleman dice que "tenemos dos mentes, una que piensa y otra que siente" Otra manera de entenderlo es que el pensamiento es un proceso con muchas caras. Las emociones son una de las facetas de ese proceso, una parte tan integral del mismo como el pensamiento lógico, lineal y verbal del hemisferio izquierdo. De la misma manera que no pensamos sólo con un único hemisferio, sino que los dos son necesarios, tampoco nos limitamos a procesar la información, además la sentimos.

A la hora de andar por la vida es más importante saber descifrar nuestras emociones que saber despejar ecuaciones de segundo grado. Las empresas lo saben bien y cuando contratan a alguien no piden sólo un buen currículo, además buscan un conjunto de características psicológicas como son la capacidad de llevarse bien con los colegas, la capacidad de resolver conflictos, la capacidad de comunicarse, etc. El 
que tengamos 0 no esas cualidades 0 habilidades va a depender del grado de desarrollo de nuestra inteligencia emocional.

Cuando hacemos un examen de poco nos sirve saber las respuestas si nos ponemos tan nerviosos que no somos capaces de contestar las preguntas adecuadamente. Naturalmente tampoco es suficiente estar tranquilo, hay que saber las respuestas del examen y saber mantener la calma.

Pero mientras que normalmente pasamos mucho tiempo aprendiendo (y enseñando) las respuestas del examen no solemos dedicarle ni un minuto a aprender (o enseñar) cómo controlar los nervios o cómo calmarlos.

Nuestro sistema educativo no es neutro, no le presta la misma atención a todos los estilos de aprendizaje, ni valora por igual todas las inteligencias o capacidades. No hay más que mirar el horario de cualquier escolar para darse cuenta de que la escuela no le dedica el mismo tiempo a desarrollar la inteligencia corporal - kinestésica y la inteligencia lingüística, por poner un ejemplo.

En cuanto a la inteligencia emocional (la capacidad de entender y controlar las emociones) la escuela simplemente la ignora. No es tanto que no la considere importante, es que su aprendizaje se da por supuesto.

El colegio no hace más que reflejar la visión de la sociedad en su conjunto. A nadie le extraña que un alumno tenga que hacer muchos ejercicios para aprender a resolver ecuaciones, sin embargo, no nos planteamos la necesidad de adiestrar a nuestros alumnos en como prestar atención durante una conversación, por ejemplo, o concentrarse como lo hacen en la cultura oriental.

Naturalmente, además, no sabemos como hacerlo. Mejor dicho, porque nunca lo hemos considerado parte de nuestra tarea no hemos aprendido a hacerlo. Lo que se está planteando ahora por primera vez es que, de la misma manera que practicamos y desarrollamos la capacidad de escribir o la capacidad de hacer deporte podemos desarrollar y practicar el conjunto de capacidades que nos permiten relacionarnos de manera adecuada con el mundo exterior y con nosotros mismos, es decir la inteligencia emocional. (2)

\section{3. ¿UNA O MUCHAS?}

Gardner ha declarado que cuando formuló en 1983 la teoría de las inteligencias múltiples, encontró poca acogida entre sus compañeros de profesión: "Mi teoría gustó a unos cuantos psicólogos, desagradó a unos pocos más y la mayoría la ignoró", (1995; 14). Un rasgo llamativo de esta situación es que cuando ya se encontraba convencido de que su proposición estaba condenada al olvido, como tantas otras en la historia de la disciplina, inesperadamente comenzó a recibir una gran atención de los educadores: "Existía otro público con un auténtico interés por mis ideas: el público de los profesionales de la educación", (1995; 15).

Este episodio no es meramente anecdótico. Detrás de este hecho late una cuestión de carácter epistemológico que merece un comentario. La teoría no recibió en ningún momento una aprobación al interior de la disciplina en que se originó, ya sabemos que los miembros del ámbito la ignoraron. Ni siquiera fue debatida en forma amplia y rigurosa. Sin embargo, despertó un interés positivo en otro ámbito, y 
rápidamente comenzó a ser consumida y aplicada, lo que derivó en la aparición de nuevas prácticas pedagógicas e institucionales.(3)

Lamentablemente, a pesar de su valor intrínseco y sus perspectivas de aplicación, la teoría de Howard Gardner se presenta como una propuesta no totalmente clara desde el punto de vista científico. Si bien muchos de los argumentos gardnerianos sostenidos en su libro Estructuras de la mente (1994) parecen coherentes y siguen las propuestas planteadas por otros científicos; hay algunos puntos oscuros en la misma.

El constructo de inteligencia propuesto por Gardner supone -entre otros criterios- la existencia de un correlato neurofisiológico que justifique la existencia de ellas. De esta manera cada una de las ocho inteligencias, está ubicada en un lugar determinado de la corteza cerebral y -si este llegara a dañarse- se evidenciaría en una disminución de dicha capacidad. Su postura está muy acorde con algunos descubrimientos llevados a cabo en el campo de la neurobiología que parecieran indicar la posible localización de ciertas funciones en el cerebro. Así por ejemplo, se sabe que el hemisferio izquierdo está más relacionado con las capacidades lingüísticas, mientras el derecho lo esta con las espaciales y musicales. No obstante, la total ubicación de cada inteligencia, en los términos que Gardner utiliza, es todavía incierta.

Existen otros puntos teóricos en los cuales la propuesta de Gardner ofrece puntos flacos. Uno de ellos puede ser la noción de modularidad, que sirve para fundamentar la total independencia de cada capacidad. Dicha noción fue tomada en préstamo de la propuesta de Jerry Fodor, quien sostiene la existencia de ciertas capacidades modulares que constituyen la mente humana, como el lenguaje o la percepción. No obstante, la noción gardneriana guarda poca o ninguna relación con la descripción inicial que este autor realiza de los módulos de la mente. Para Fodor ellos funcionan como mecanismos automáticos y predeterminados, mucho más fijos y ciegos que las inteligencias propuestas por Gardner.

Pero sobre todo, en lo que las ideas de Gardner parecen ser más frágiles es en la medición de cada una de estas inteligencias en la actuación real de cada sujeto.

Ahora bien, ¿cómo puede medirse habilidades "teóricamente separadas", pero "realmente" tan integradas unas con otras? Uno de los instrumentos de medición desarrollados en los últimos años, fue el MIDAS (Multiples Intelligence Development Assesment Scales) creado por el Dr. Branton Shearer del Multiple Intelligence Research and Consulting, de Ohio. El MIDAS es una entrevista en la cual el sujeto se refiere a sus habilidades y preferencias y a partir de esa información -corroborada a veces por padres 0 maestros- se observa la distribución de cada habilidad. Como parte de una investigación llevada a cabo en el seno de la Universidad Católica de Valparaíso, dicho instrumento fue traído a Chile en 1995 y traducido al español.

Al analizarse los puntajes pudo verse que las distintas inteligencias se encontraban altamente correlacionadas entre sí. En otras palabras, un individuo tendía a tener puntajes igualmente altos e igualmente bajos en casi todas las capacidades rotuladas como inteligencias. Dicha interrelación de los factores, no permite hablar de una independencia tan radical como Gardner la propone. Más bien podría hablarse de grandes tendencias generales que parecen evidenciarse en la conducta del sujeto. En otras palabras, sea cual sea el estatus teórico de la teoría de Gardner, su medición empírica carece de claridad. 
$\mathrm{Si}$ bien es posible hablar de habilidades humanas diferentes, es necesario cuestionarse las afirmaciones últimas de esta propuesta: ¿son dichas habilidades tan independientes y autónomas como Gardner sostiene? ¿Pueden considerarse con una importancia tan medular para la actividad intelectual al punto de ser denominadas inteligencias? (4)

\section{4. ¿QUÉ DIFICULTADES OFRECE?}

Todo este andamiaje de las inteligencias múltiples trae a la realidad unas aplicaciones que no se pueden dejar pasar por alto ya que a la vez afectan al alumno y al maestro.

- Implantar estas ideas dentro de un currículo tradicionalista no es fácil de lograr.

- Necesidad de adiestramiento en servicio.

- El programa de clases y los horarios rígidos han de mortificarse para darle paso a horarios más flexibles.

- La necesidad de tiempo extra para preparar lecciones y materiales didácticos.

- Necesidad de más personal docente en algunas escuelas.

- La necesidad de un currículo que tenga al estudiante como centro del proceso enseñanza aprendizaje y que este sea considerado como un individuo.

- El alumno tiene que prepararse para que pueda trabajar con estos nuevos enfoques.

- El estudiante ha de evaluarse en forma distinta a la que está acostumbrado.

- El uso de las inteligencias múltiples como herramienta de instrucción.

- Necesidad de más tiempo para que el estudiante pueda trabajar en una forma diferente a la acostumbrada.

- Temor de ponerle "sellos" al estudiante.

- Uso de la tecnología y materiales concretos.

Frente al inicio de un nuevo milenio, éstas ideas presentan un reto a los educadores. ¿Se sigue dando vueltas en la búsqueda de nuevas alternativas sin detenerse a pensar o se agilizan formas "atrevidas", con los pies puestos en tierra, que puedan ayudar a formar un individuo que en verdad pueda ser útil a su familia, a su comunidad y a la sociedad en que vive? Todo cambio en la educación tiene que contar con el maestro de la sala de clases y lógicamente con el alumno que es el centro de todo proceso educativo.

Si la inteligencia es la capacidad que le permite al ser humano resolver problemas, ¿por qué no le brindamos a éste la oportunidad de desarrollarla a plenitud en la medida que lo permita su condición particular? (5)

\section{5. ¿CÓMO EMPEZAR?}

Con antelación a la aplicación de cualquier modelo de aprendizaje basado en las inteligencias múltiples, debemos en primera instancia aplicárnoslo a nosotros mismos como educadores y estudiantes 
adultos, porque si no tenemos una comprensión de la teoría íntimamente ligada a la experiencia y hayamos hecho nuestro este conocimiento, es decir estemos en condiciones de aplicarlo, no como copia, sino como modelo propio, no podremos trasmitirlo con éxito.

Por lo tanto el primer paso es determinar la naturaleza y calidad de nuestras propias inteligencias múltiples y buscar las maneras de desarrollarlas en nuestras propias vidas. Cuando nos abocamos a esta tarea se pondrá de manifiesto como nuestra particular fluidez o falta de ella afecta nuestras competencias como educadores. Esta no es una tarea fácil por cuanto no existe una herramienta de medición que nos asegure cual es el grado o el cociente alcanzado en cada una de las inteligencias, por lo que debemos ampliar nuestro campo de observación y a través de una evaluación realista de sus desempeños en las muchas clases de actividades, tareas y experiencias que se asocian con cada inteligencia es que obtendremos indicadores sobre el nivel alcanzado en cada una de ellas.

Esta teoría es una herramienta especialmente útil para observar nuestras fortalezas y debilidades en las áreas que utilizamos los docentes, porque nos permite observar todas las actividades que realizamos para alcanzar nuestros objetivos, y también cuales acciones dejamos de lado por cuanto no nos sentimos cómodos al ejecutarlas.(6)

Desarrollar hasta un grado aceptable de competencia cada una de las inteligencias, depende según Armstrong de tres factores principales:

- Dotación biológica, incluyendo los factores genéticos o hereditarios, y los daños o heridas que el cerebro haya podido recibir antes, durante o después del nacimiento.

- Historia de la vida personal, incluyendo las experiencias con los padres, docentes, pares, amigos y otras personas que ayudan a hacer crecer las inteligencias o las mantienen en un bajo nivel de desarrollo.

- Antecedente cultural o histórico, incluyendo la época y el lugar donde uno nació y se crió, y la naturaleza y estado de los desarrollos culturales o históricos en diferentes dominios.

\section{6. ¿CÓMO PLANIFICAR?}

Haciendo un diagnóstico de las potencialidades de los niños y teniendo en cuenta esta grilla, podremos seleccionar las actividades a realizar.

\begin{tabular}{|c|l|l|l|}
\hline & \multicolumn{1}{|c|}{ DESTACA EN } & \multicolumn{1}{c|}{ LE GUSTA } & \multicolumn{1}{c|}{ APRENDE MEJOR } \\
\hline $\begin{array}{c}\text { AREA LINGüíSTICO- } \\
\text { VERBAL }\end{array}$ & $\begin{array}{l}\text { Lectura, escritura, } \\
\text { narración de historias, } \\
\text { memorización de } \\
\text { fechas, piensa en } \\
\text { palabras }\end{array}$ & $\begin{array}{l}\text { Leer, escribir, contar } \\
\text { cuentos, hablar, } \\
\text { memorizar, hacer } \\
\text { puzzles }\end{array}$ & $\begin{array}{l}\text { Leyendo, escuchando } \\
\text { y viendo palabras, } \\
\text { hablando, escribiendo, } \\
\text { discutiendo y } \\
\text { debatiendo }\end{array}$ \\
\hline $\begin{array}{c}\text { LÓGICA - } \\
\text { MATEMÁTICA }\end{array}$ & $\begin{array}{l}\text { Matemáticas, } \\
\text { razonamiento, lógica, } \\
\text { resolución de } \\
\text { problemas, pautas. }\end{array}$ & $\begin{array}{l}\text { Resolver problemas, } \\
\text { cuestionar, trabajar } \\
\text { con números, } \\
\text { experimentar }\end{array}$ & $\begin{array}{l}\text { Usando pautas y } \\
\text { relaciones, } \\
\text { clasificando, } \\
\text { trabajando con lo } \\
\text { abstracto }\end{array}$ \\
\hline ESPACIAL & $\begin{array}{l}\text { Lectura de mapas, } \\
\text { gráficos, dibujando, } \\
\text { laberintos. nuzzles. }\end{array}$ & $\begin{array}{l}\text { Diseñar, dibujar, } \\
\text { construir, crear, soñar } \\
\text {... } .\end{array}$ & $\begin{array}{l}\text { Trabajando con dibujos } \\
\text { y colores, } \\
\text { visualizando. usando }\end{array}$ \\
\hline
\end{tabular}




\begin{tabular}{|c|c|c|c|}
\hline & $\begin{array}{l}\text { imaginando cosas, } \\
\text { visualizando }\end{array}$ & despierto, mirar dibujos & $\begin{array}{l}\text { su ojo mental, } \\
\text { dibujando }\end{array}$ \\
\hline $\begin{array}{l}\text { CORPORAL - } \\
\text { KINESTÉSICA }\end{array}$ & $\begin{array}{l}\text { Atletismo, danza, arte } \\
\text { dramático, trabajos } \\
\text { manuales, utilización } \\
\text { de herramientas }\end{array}$ & $\begin{array}{l}\text { Moverse, tocar y } \\
\text { hablar, lenguaje } \\
\text { corporal }\end{array}$ & $\begin{array}{l}\text { Tocando, moviéndose, } \\
\text { procesando } \\
\text { información a través de } \\
\text { sensaciones } \\
\text { corporales. }\end{array}$ \\
\hline MUSICAL & $\begin{array}{l}\text { Cantar, reconocer } \\
\text { sonidos, recordar } \\
\text { melodías, ritmos }\end{array}$ & $\begin{array}{l}\text { Cantar, tararear, tocar } \\
\text { un instrumento, } \\
\text { escuchar música }\end{array}$ & $\begin{array}{l}\text { Ritmo, melodía, cantar, } \\
\text { escuchando música y } \\
\text { melodías }\end{array}$ \\
\hline INTERPERSONAL & $\begin{array}{l}\text { Entendiendo a la } \\
\text { gente, liderando, } \\
\text { organizando, } \\
\text { comunicando, } \\
\text { resolviendo conflictos, } \\
\text { vendiendo }\end{array}$ & $\begin{array}{l}\text { Tener amigos, hablar } \\
\text { con la gente, juntarse } \\
\text { con gente }\end{array}$ & $\begin{array}{l}\text { Compartiendo, } \\
\text { comparando, } \\
\text { relacionando, } \\
\text { entrevistando, } \\
\text { cooperando }\end{array}$ \\
\hline INTRAPERSONAL & \begin{tabular}{|l|} 
Entendiéndose a sí \\
mismo, reconociendo \\
sus puntos fuertes y \\
sus debilidades, \\
estableciendo objetivos
\end{tabular} & $\begin{array}{l}\text { Trabajar solo, } \\
\text { reflexionar, seguir sus } \\
\text { intereses }\end{array}$ & $\begin{array}{l}\text { Trabajando solo, } \\
\text { haciendo proyectos a } \\
\text { su propio ritmo, } \\
\text { teniendo espacio, } \\
\text { reflexionando. }\end{array}$ \\
\hline NATURALIST A & $\begin{array}{l}\text { Entendiendo la } \\
\text { naturaleza, haciendo } \\
\text { distinciones, } \\
\text { identificando la flora y } \\
\text { la fauna }\end{array}$ & $\begin{array}{l}\text { Participar en la } \\
\text { naturaleza, hacer } \\
\text { distinciones. }\end{array}$ & $\begin{array}{l}\text { Trabajar en el medio } \\
\text { natural, explorar los } \\
\text { seres vivientes, } \\
\text { aprender acerca de } \\
\text { plantas y temas } \\
\text { relacionados con la } \\
\text { naturaleza }\end{array}$ \\
\hline
\end{tabular}

Cuadro traducido por Nuria de Salvador de Developing Students' Multiple Intelligences.

NichOLson-NeLson, K. ( New York: Scholastic Professional Books 1998).

Por otra parte, Armstrong, en su libro Las inteligencisas múltiples en el aula, muestra un la forma de planificar y llevar adelante clases sobre la base de IM, propone variados ejercicios, modelos de clases y una excelente información para ayudar al docente. 
Este es un ejemplo de esquema de planificación que propone:

$$
\begin{aligned}
& \text { Discutir la lógica de } \\
& \text { Los signos de puntuación }
\end{aligned}
$$

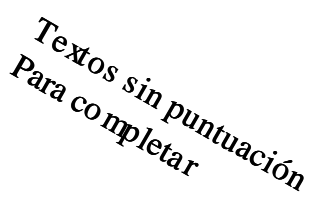

Escribir sus propias
oraciones

$$
\text { LINGÜÍSTICO } \hat{\lambda}_{\hat{a}}
$$

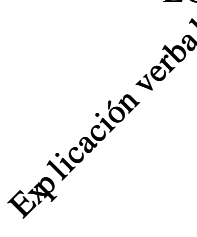

LÓGICO-MATEMÁTICO Sustituir los signos duación por números

\section{OBJETIVO}

MUSICAL

i pudiera ser un igno de puntuación..."

Tarjetas con los signos án años
Para jugar con 10 compaños

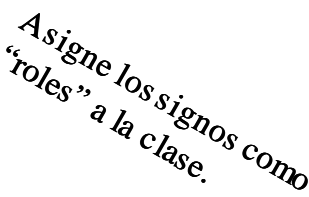

INTERPERSONAL

La función de los signos de puntuación

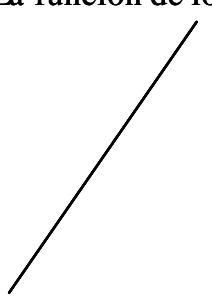

$J_{u_{e_{0}} \text { social }_{\mathrm{C}_{\text {al }}}}$

Sustituir los signos por animales o plantas

\section{NATURISTA}

ESPACIAL

$$
\text { convertir en imágenes }
$$

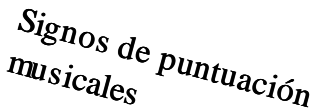$$
\text { Actúe los signos }
$$

\section{CORPORAL-KINÉTICO}

Marcar los signos en las
Espaldas de los compaños

Obra teatral de
Toda la clase

\section{7. ¿DÓNDE SE APLICA?}

Según Fernando Lapalma, ya existen instituciones educativas trabajando con las inteligencias múltiples aquí y en otros países. Estados Unidos, tanto a nivel privado como a nivel estatal, (con sus escuelas Key y otros proyectos como Spectrum, para nivel inicial y Arts Propel para nivel medio), Canadá, Israel, Venezuela, Italia, Australia, Nueva Zelanda, entre otros son los que han tomado la delantera en este cambio. Siendo en algunos de ellos ya oficial su aplicación.

Luego de diez años de aplicación quedan como corolario los siguientes:

Minimización de los problemas de conducta

Aumento de la autoestima

Desarrollo de la cooperación 
Incremento del número de líderes positivos

Crecimiento del interés y afecto por la escuela y el estudio

Presencia constante del humor

Incremento del conocimiento en un $40 \%$ (7)

\section{CONCLUSIONES}

En nuestra realidad educativa, no todo es válido ni todo es equivocado, en las reformas educativas latinoamericanas se deben conocer las distintas teorías y experiencias educativas en el mundo para poder reformularlas o adaptarlas a nuestras necesidades.

Independientemente de la polémica de considerar "inteligencias", "capacidades" o "fortalezas" a esas facultades más o menos desarrolladas en las personas, a los docentes nos resulta de suma utilidad diagnosticarlas en nuestros alumnos, ya que nos permite comprenderlos más y delinear las actividades más apropiadas para obtener los máximos aprovechamientos. Claro que para eso debemos informarnos, recibir ayuda, disponer de tiempo extra, institucionalizar el trabajo y comprometer a toda la comunidad. Tarea para nada fácil pero no imposible.

El docente intuitivamente ya hace adecuaciones y actividades variadas y especiales, falta fundamentarlas, sistematizarlas, incorporarlas a la tarea diaria y, a la hora de evaluar tenerlas en cuenta. No podemos sólo hacerlos cantar y bailar y después evaluarlos por escrito.

Por otra parte, debemos tratar de desarrollar las facultades que no lo están y creo que allí está el mayor desafío. La capacidad de inventiva y creatividad, siempre puesta de manifiesto por los docentes, sólo necesita ser "activada" por un estímulo que bien puede ser éste.

Si seguimos encontrando culpables fuera de nosotros mismos y no buscamos las formas de cambiarnos y cambiar a nuestros alumnos, no hay futuro para los países latinoamericanos.

\section{NOTAS}

1- Extractado de: Ricardo López Pérez. Revista Enfoques Educacionales Vol. I №2 1998Departamento de Educación. Facultad de Ciencias Sociales. Universidad de Chile.

2- $\quad$ Extractado de un artículo publicado por Fernando Lapalma, autor del proyecto íMPETU y de diferentes cursos y seminarios sobre el tema.

3- $\quad$ Del trabajo de Ricardo López Pérez.

4- $\quad$ Extractado de la Tesis doctoral en Lingüística Aplicada de Nina Crespo Allende. Universidad de Chile. Facultad de Ciencias Sociales.

5- De la Conferencia pronunciada en el SIMPOSIO INTERNACIONAL DE EDUCACIÓN EN LA DIVERSIDAD, celebrado en panamá, del 28 al 30 de enero de 2000 por Magíster Joaquín Padovani, Departamento de Matemáticas y Ciencias Aplicadas. Universidad Interamericana de Puerto Rico.

6- Extractado del Seminario de Inteligencias múltiples. Lic. Miguel Lupiañez Universidad Champagnat, Mendoza, Argentina.

7- $\quad$ Del trabajo de Fernando Lapalma 


\section{BIBLIOGRAFÍA}

ARMSTRONG, Thomas. Las inteligencias múltiples en el aula. Manantial.1999

BORSANI, María José. Adecuaciones curriculares. Novedades Educativas. 2001

CRESPO ALLENDE, Nina. Inteligencias múltiples y Aprendizajes Escolares. http://rehue.csociales.uchile.cl/rehuehome/facultad/publicaciones/Talon/talon5/

Crespo Allende, Nina. Tesis Doctoral en Lingüística Aplicada. Universidad de Chile, Facultad de Ciencias Sociales

GARDNER, Howard. Estructuras de la mente. Fondo de Cultura económica. México.1994.

LAPALMA, Fernando. Inteligencias Múltiples. www.galeon.hispavista.com/aprenderaaprender/intmultiples/lapalma.htm

LÓPEZ PÉREZ, Ricardo. En torno a Inteligencias Múltiples. Revista Enfoques Educacionales Vol. I № 21998. Departamento de Educación, Facultad de Ciencias Sociales. Universidad de Chile.

LUPIAÑEZ, Miguel. Inteligencias Múltiples y desarrollo personal. http://www.uch.edu.ar/rrhh/temasVarios/Psicología/Inteligencias Multiples.

PADOVANI, Joaquín. Implicaciones para maestros y estudiantes. AMEI. http://www.waece.com

Revista Novedades Educativas № 123 Marzo 2001.

\section{OTROS SITIOS RECOMENDADOS}

Juego didáctico basado en Inteligencias Múltiples

Juego Didáctico. Fracciones Mágicas de AutoMind. !Nunca pensé que era tan

fácil. 1. Composición de Piezas: El juego tiene dos conjuntos de piezas: ...

http://www.automind.cl/FRAC.HTM

\section{INTELIGE}

Aplicando Inteligencias Múltiples. ANTECEDENTES DEL CURSO. 1.- NOMBRE DEL CURSO: Aplicando Inteligencias Múltiples. 2.-FUNDAMENTACIÓN ... http://www.automind.cl/INTELIGE.HTM

Test de Inteligencias Múltiples

Test de Inteligencias Múltiples Esta herramienta ha sido adaptada de la obra de Howard Gardner en el tema de inteligencias múltiples e incluye una ... http://www.fleming.edu.pe/spn/testintelig.htm 


\title{
Contactar
}

Revista lberoamericana de Educación

\author{
Principal OEI
}

